\title{
The circRNA-MYLK plays oncogenic roles in the Hep-2 cell line by sponging microRNA-145-5p
}

\author{
Yao Chen, Yanmei Wang, Congcong Li, Xuechang Li, Tiejun Yuan, Shuqin Yang \\ and Xiaoyan Sun
}

Department of Otolaryngology, Weifang People’s Hospital, Weifang, China

\begin{abstract}
For the exploration of circular RNA light chain kinase (circRNA-MYLK), siRNA\#1 and siRNA\#2 targeting circRNA-MYLK as well as microRNA(miR)-145-5p inhibitor were transfected. Viability was valued with the CCK-8. The protein expression was examined relying on Western blot. The expression of circRNA-MYLK or miR-145-5p was tested depending on qRT-PCR. The apoptotic/ migration/invasion rate was separately measured by the Annexin v-FITC/PI with flow cytometer or chambers assays. CircRNA-MYLK was overexpressed in tumor tissue. Silencing circRNA-MYLK induced the inhibitions of viability, invasion and migration, as well as the blocks of MEK/ERK and NF- $\kappa B$ cascades, however, silencing circRNA-MYLK led to provoking of apoptosis. Besides, circRNA-MYLK silencing stimulated the over-production of miR-145-5p, whose silencing abolished the effects of siRNA\#1 and siRNA\#2 of circRNA-MYLK on those factors above. The circRNA-MYLK had oncogenic roles via targeting miR-145-5p in the Hep-2 cell line via stimulating MEK/ERK and $\mathrm{NF}-\kappa \mathrm{B}$ cascades
\end{abstract}

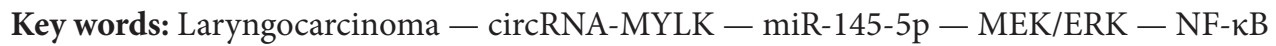

\section{Introduction}

Laryngocarcinoma is considered as one of the most widespread tumors, and it is responsible for the high incidences mortality of tumor diseases (Lampri et al. 2015; Li et al. 2016). There are some treatment methods for this disease including the surgery, chemo-radiotherapy as well as utilization of Chinese herbs (Lambert et al. 2010; Liu et al. 2010). Nonetheless, they are restrained by poor efficiency or obvious side effects (Miao et al. 2013). Recently, numerous studies have revealed that non-coding RNAs (ncRNAs) serve essential actions in the expanding of laryngocarcinoma (Chen et al. 2018). However, the pathogenesis of laryngocarcinoma and the potential mechanism of ncRNAs have not been elucidated fully.

Lots of researches demonstrated that circular RNAs (circRNAs) could control cellular reactions and tumor progression (Chen et al. 2015, 2017). Sun and the colleagues stated

Correspondence to: Xiaoyan Sun, Department of Otolaryngology, Weifang People's Hospital, No. 151 Guangwen Street, Kuiwen District, Weifang 261000, Shandong, China

E-mail: xiaoyan30s@163.com that circRNAs had vital characters in the tumorigenesis of laryngeal squamous cell carcinomas (LSCC) and had potentials to function in the diagnosis and development of LSCC (Xuan et al. 2016). Moreover, it has been reported that circRNA-myosin light chain kinase (MYLK), which is derived from the MYLK gene, is over-expressed in breast cancer (BC), being connected with the stage and grade of BC (Zhong et al. 2017). What's more, in hepatocellular carcinoma (HCC) experiment, circRNA-MYLK targeted the microRNA-362-3p (miR-362-3p)/Rab23 axis and thus to be positively related to the occurrence and expansion of this tumor (Li et al. 2019). More important, circRNA-MYLK was beneficial to the survival of prostate cancer (PCa) cells by adjusting the proliferation and migration procedures (Dai et al. 2018), indicating the possibilities that circRNA-MYLK got involved in the progression of tumors.

Over the past decades, miRNAs have been thoroughly identified as key regulators in diverse tumors, playing oncogenic or anti-tumor effects through their downstream genes or signaling cascades. For example, miR-451a was downregulated in papillary thyroid cancers, in which abundant miR-451a could contribute to cancer cells apoptosis but 
inhibit the proliferation and epithelial-mesenchymal transition (Fan et al. 2019). miR-362-3p could be a prognostic biomarker for cervical adenocarcinoma (ADC) and squamous cell carcinoma (SCC) through an regulatory network (Song et al. 2019). The miR-29a played crucial roles in the drug-sensitivity of NSCLC cells and was connected with tumor-node-metastasis classification (Chen et al. 2019). Importantly, the miR-145-5p had some connections with the functional mechanism of taurine up-regulated 1 (TUG1) in laryngocarcinoma. Generally TUG1 was conductive to the enhancement of laryngocarcinoma through silencing miR145-5p (Zhuang et al. 2019). The direct modulation of the circRNA-MYLK and miR-145-5p is still uncertain.

In this current experiment, we wondered how circRNAMYLK served in laryngocarcinoma in vitro leaning on the Hep-2 cell line.

\section{Materials and Methods}

\section{Ethics approval and consent to participate}

All procedures performed in studies involving human participants were in accordance with the ethical standards of the institutional committee and with the 1964 Helsinki declaration and its later amendments or comparable ethical standards. We obtained informed consents from every patient, and our research was with the permission of the Medical Ethics Committee of the Weifang People's Hospital.

\section{Clinical specimens}

These material tissues were obtained from the patients of Weifang Hospital (Weifang, China) who never got treatment before. Besides, our present research was performed with the permission of every patient and the Medical Ethics Committee of the Weifang Hospital.

\section{Cell cultivation}

Hep-2 cell line from the ATCC (Manassas, Virginia, USA) was considered as the experimental object. Those cells were cultivated through the Dulbecco's modified Eagle medium (DMEM) medium (BD Biosciences, San Jose, CA, USA), including $10 \%$ serum as well as growth factors (all from Gibco, Waltham, Massachusetts, USA), at $37^{\circ} \mathrm{C}$, until $80-90 \%$ attachment was observed.

\section{Cell transfection}

Small-interfering RNAs were introduced to disturb the production of circRNA-MYLK, which was individually called siRNA\#1 and siRNA\#2. The siRNA\#1, siRNA\#2 and the miR-145-5p inhibitor (Sangon Biotech, Shanghai, China) were individually synthesized to induce alterations of circRNA-MYLK and miR-145-5p in Hep-2 cell line. Reagent of lipofectamine 3000 (Roche, Basel, Switzerland) helped to perform this transfection experiment. After three days, we harvested the highest efficiency. In addition, the nucleotide sequences of siRNA\# 1 and siRNA\#2 were as following, siRNA\#1: 5'-ACT ACAGGTATGGCCTCACAA-3'; siRNA\#2: 5'-TACAGGTATGGCCTCACAAGT-3'.

\section{Cell viability assessing}

Cell viability was assessed through the CCK-8. In the beginning, Hep-2 cells were divided into different groups to undergo diverse stimulus at $37^{\circ} \mathrm{C}$. After that, the CCK-8 was injected and mixed with those Hep-2 cells for another $1 \mathrm{~h}$. Based on that, the suspension absorbance of Hep-2 cell line was assessed leaning on a microplate reader (Bio-Rad, Hercules, California, USA).

\section{Apoptosis assay}

Totally, those treated Hep- 2 cells, which had been adjusted and cultivated in a 6-well plate, were washed and collected. Besides, adherent cells were digested by means of trypsin (Polyplus transfection, Strasbourg, Bas-Rhin, France). With the re-suspension of those cells, Annexin V-FITC and propidium iodide (PI) were mixed to incubate with the cells for 10-16 min for flow cytometer detecting avoiding light.

\section{Migration and invasion assay}

The migratory rate of Hep-2 cell line was checked by a twochamber assay. Followed by a classical nurturing, the upper chamber was filled with cells suspension and the lower chamber was filled with the normal medium (BD Biosciences). After washing, cells were subsequently treated by methanol (Beyotime Biotechnology, Shanghai, China) and crystal violet (Sigma-Aldrich, St. Louis, Missouri, USA) (10-20 min). In the end, we counted the migrated cells under a Canon microscope (Tokyo, Japan) depending on five fields that were randomly selected. Additionally, here, the invasive capability of cells was assessed by a similar method. Generally, the participation of the matrigel glue (Millipore) was the only difference between migration detection and the invasion valuation.

Quantitative reverse transcription polymerase chain reaction $(q R T-P C R)$

By means of lysing function of trizol reagent (Solarbio, Beijing, China) and the protective effects of DNaseI (Promega, 
Madison, wisconsin, USA), total RNA were extracted from Hep-2 cells. Furthermore, the SuperScript ${ }^{\mathrm{TM}}$ III kit (Solarbio) and Taqman Master series kits (Hyclone, Logan, UT, USA) were introduced to the qRT-PCR assay. We took $\beta$-actin or $\mathrm{U} 6$ as the internal reference. The corresponding primer sequences were including circRNA-MYLK: Forward 5'-CAGTGCATGCTGTTTGTTCA-3', Reverse 5'-TCGGAGCCTTGACTTCCAG-3'; U6: Forward 5'-CTCGCTTCGGCAGCA CA-3', Reverse 5'-AACGCTTCACGAATTTGCGT-3'; miR-145-5p: Forward 5'-ACACTC CAGCTGGGGTCCAGTTTTCCCAGG A-3', Reverse 5'-TGGTGTCG TGGAGTCG-3'; GAPDH: Forward 5'-TATGATGATATCAAGAGGGTAG T-3', Reverse 5-TGTATCCA AACTCATTGTCATAC-3.

\section{Western blot}

We prepared the RIPA buffer (Beyotime Biotechnology) at a $4^{\circ} \mathrm{C}$ atmosphere in advance. Once the proteins were isolated from cells, the protease inhibitor (Qiagen, Hilden, Germany) was utilized for protein protection. Those target proteins were transferred into a PVDF membrane (Hyclone) by the way of electrophoresis. The primary antibodies used here consisted of anti-Bcl-2 (ab32124, Abcam, Cambridge, UK), anti-Bax (ab32503), anti-cleaved-caspase 9 (ab2324), anti-matrix metalloproteinase (MMP) 2 (ab37150), anti-cleaved-caspase 3 (ab2302), anti-MMP 9 (ab73734), anti-inhibitor of metalloproteinase 1 (TIMP-1) (ab38978), anti-vimentin (ab92547), anti-mitogen-activated protein kinase (MAPK)/extracellular regulated protein kinases (ERK) kinase (MEK) (ab215263), anti-p-MEK (ab194754), anti-p-ERK (ab201015), anti-ERK (ab17942), anti-p-NF-kB-p65 (ab222494), anti-nuclear factor kappa-B (NF- $\mathrm{B}$ )-p65 (ab207297) and anti- $\beta$-actin (ab16039). All of them were attached to the target protein bands at $4^{\circ} \mathrm{C}$ overnight, before the goat anti-rabbit (HRP) (ab7090) was introduced to the membrane at $25^{\circ} \mathrm{C}(1 \mathrm{~h})$. Image Lab ${ }^{\text {Tw }}$ Software (Bio-Rad) was responsible for protein bands quantification.

\section{Statistical analysis}

The experimental analysis could be attributed to the SPSS (19.0), showing as the mean \pm standard deviation (SD). $t$-test or analysis of variance (ANOVA) was responsible for $p$ values, which less than 0.05 was adopted with statistically significant.

\section{Results}

CircRNA-MYLK is highly expressed in tissues of patients with laryngeal cancer

The RNA expression of circRNA-MYLK was tested leaning on qRT-PCR. By contrast, the circRNA-MYLK had higher production level in the tumor tissue ( $p<0.001$, Fig. 1A). Moreover, the siRNA\# 1 and siRNA\#2 of circRNA-MYLK were produced and transfected into the Hep-2 cells. According to the detection outcomes, the production of the circRNA-MYLK was significantly constrained by the siRNA\#1 (59\%) and siRNA\#2 (70\%) (both $p<0.001$, Fig. 1B). This data told that the over-production vectors were successfully constructed in the Hep-2 cells.

\section{Knockdown of circRNA-MYLK inhibits contributes to cell survival}

The cell viability was separately and significantly decreased about $28 \%$ or $37 \%$ due to the transfections of siRNA\# 1 and siRNA\#2 (both $p<0.05$, Fig. 2A). Additionally, the apoptotic rates (2.8 or 2.2 times) (both $p<0.001$, Fig. $2 \mathrm{~B}$ ) and the generation of Bax (1.9 or 2.5 times), cleaved caspase 3 ( 3 or 4.5 times) and cleaved caspase 9 (3.5 or 3 times) were significantly stimulated with the inhibition of circRNAMYLK (all $p<0.001$ ), while the Bcl-2 was significantly and individually reduced about 61 or $57 \%$ (both $p<0.001$, Fig. $2 \mathrm{C}, \mathrm{D})$. These results displayed that circRNA-MYLK was probably conductive to the viability but prevented apoptosis in Hep-2 cells.

\section{Knockdown of circRNA-MYLK suppresses Hep-2 cells migrations and invasion}

The migration rate (36 or $41 \%$ ) (both $p<0.05$, Fig. $3 \mathrm{~A}$ ) and the invasion rate (57 or 63\%) (both $p<0.001$, Fig. $3 \mathrm{~B}$ ), as well as the productions of the MMP-2 (39 or $48 \%)$ and MMP-9 (47 or 50\%) $(p<0.05, p<0.01$ or $p<$
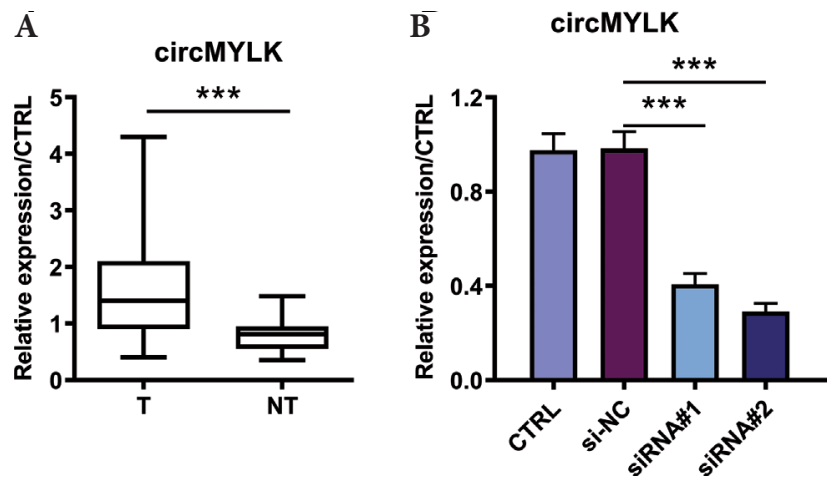

Figure 1. circRNA-MYLK was over-expressed in laryngocarcinoma tissues. After the preparation, the production level of circRNA-MYLK in laryngocarcinoma or normal tissues (A), and after the transfection of siRNA\#1 and siRNA\#2 (B), the circRNA-MYLK level were detected by qRT-PCR. ${ }^{* *} p<$ 0.001. CTRL, control; NT, none tumor; NC, negative control; T, tumor; circRNA-MYLK, circular light chain kinase; siRNA, small interfering RNA. 
A

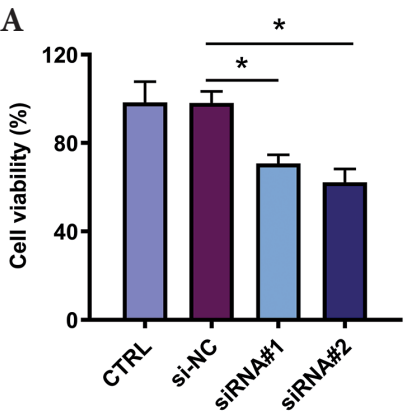

C

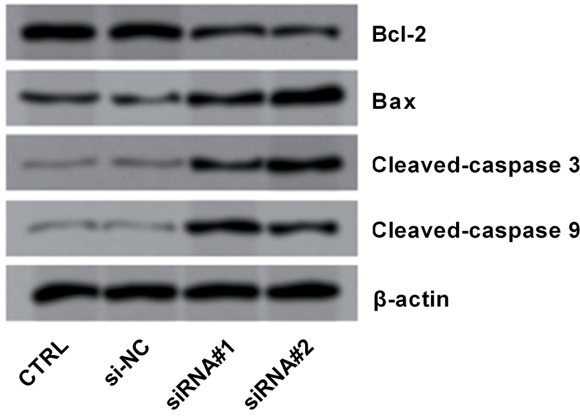

B

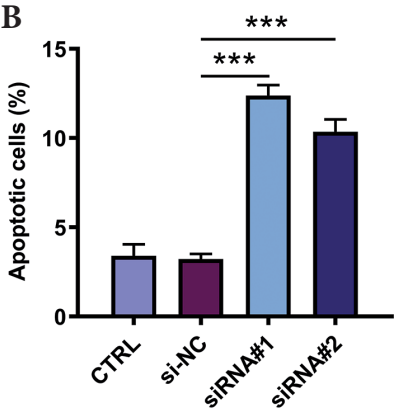

D 口 CTRL 口 SIRNA\#1

si-NC $\square$ siRNA\#2

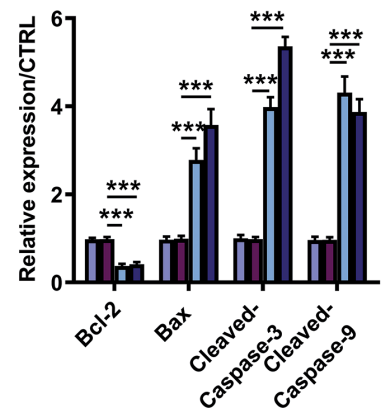

Figure 2. Knockdown of circRNA-MYLK inhibited cell viability but promoted apoptosis. After the transfection of siRNA\#1 and siRNA\#2, the cell viability (A) and the apoptotic rate (B) was explored by CCK-8 or Annexin V-FITC/PI-flow cytometer; and the apoptosis-related proteins $(\mathbf{C}, \mathbf{D})$, were examined by Western blot. ${ }^{*} p<0.05,{ }^{* * *} p<0.001$. FITC, fluorescein isothiocyanate; CCK-8, cell counting kit-8; PI, propidium iodide. For more abbreviations, see Fig. 1.
$0.001)$ and the vimentin (54 or $41 \%)(p<0.05, p<0.01)$ were extremely restrained on account of the silencing of circRNA-MYLK, excluding the TIMP-1 $(p<0.01$ or $p<$
0.001, Fig. 3C, D). All data displayed that circRNA-MYLK was probably conductive to the migrations and invasion of Hep-2 cells.
A

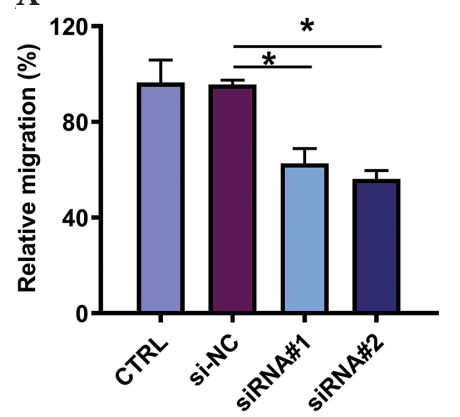

C

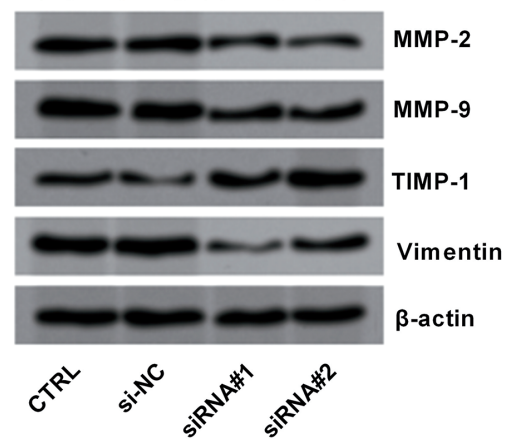

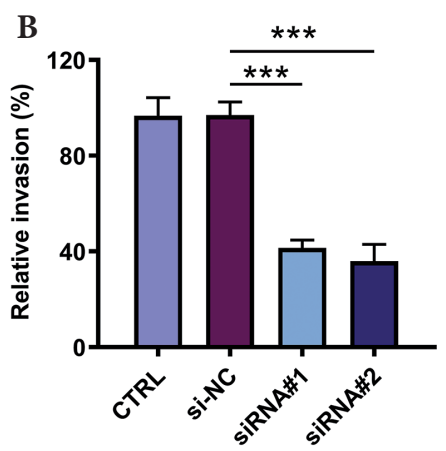

D
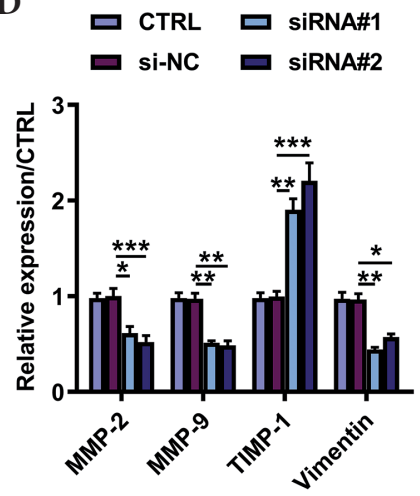

Figure 3. Knockdown of circRNA-MYLK suppresses Hep-2 cells migrations and invasion. After the transfection of siRNA\#1 and siRNA\#2, the migration rate and invasion rate were detected with migration assays $(\mathbf{A}, \mathbf{B})$ and the migration and invasion proteins were examined by Western blot (C, D). ${ }^{*} p<0.05,{ }^{* *} p<0.01,{ }^{* * *} p<0.001$. MMP 2/9, matrix metalloprotein-2/9; TIMP, tissue inhibitor of metalloprotease 1. For more abbreviations, see Fig. 1. 
Knockdown of circRNA-MYLK induces high expression of miR-145-5p

The RNA expression level of miR-145-5p was individually and remarkably elevated about 0.89 or 1.2 times by siRNA\# 1 and siRNA\#2 (both $p<0.01$, Fig. $4 \mathrm{~A}$ ). Additionally, the miR145-5p inhibitor was produced to alter the expression of the miR-145-5p in Hep-2 cells. Without question, miR-145-5p inhibitor led to an especially reduction of $69 \%$ of miR-145-5p $(p<0.01$, Fig. 4B). All data revealed that the miR-145-5p was negatively controlled by circRNA-MYLK. The miR-145-5p silent mutant was successfully constructed.

Silencing circRNA-MYLK induces apoptosis and inhibitions of cell viability, migration and invasion through up-regulating miR-145-5p

As was shown in our investigation, miR-145-5p inhibitor apparently elevated the cell viability (both 0.19 times) (both $p<0.05$, Fig. 5A). The apoptotic rate (26 or $29 \%$ ) (both $p<$ 0.05 , Fig. $5 \mathrm{~B}$ ) and productions of apoptosis-related proteins such as $\operatorname{Bax}(27$ or $40 \%)$ (both $p<0.05$ ), cleaved-caspase 3 (51 or $31 \%)(p<0.05$ or both $p<0.01)$ and cleaved caspase 9 $(60$ or $50 \%)(p<0.01$ or $p<0.001)(p<0.05, p<0.01$ or $p<0.001)$, excluding the $\mathrm{Bcl}-2$ ( 0.61 or 0.73 times) (both $p<0.05)$ were down-regulated by the miR-145-5p inhibitor (Fig. 5C, D). Furthermore, the migration rate ( 0.27 or 0.34 times) (both $p<0.05$, Fig. $5 \mathrm{E}$ ) as well as the invasion rate ( 0.37 or 0.66 times) (both $p<0.05$, Fig. $5 \mathrm{~F}$ ) of the Hep-2 cell, and the productions of these migration or invasionrelated proteins such as MMP-2 (both 0.4 times), MMP-9 ( 0.44 or 0.34 times) and vimentin ( 0.91 or 0.39 times) (all $p<0.05)$ were significantly stimulated by miR-145-5p inhibitor, which induced an decline of TIMP-1 (39 or 33\%) (both $p<0.05$, Fig. 5G, H). Those results suggested that the miR-145-5p inhibitor significantly dropped the influences of the siRNA\#1 and siRNA\#2 targeting circRNAMYLK. The miR-145-5p, which was probably a target of the circRNA-MYLK, was disadvantageous to the growth of the Hep-2 cells.

\section{CircRNA-MYLK silencing blocks MEK/ERK and NF- $\kappa B$ cascades by up-regulating miR-145-5p}

Obviously, the protein expression levels of the $\mathrm{p}-\mathrm{MEK}$ and p-ERK (Fig. 6A), as well as the p/t-MEK (44 or 55\%) and p/t-ERK (36 or 51\%) (both $p<0.05$, Fig. 6B) were significantly decreased by the siRNA\#1 or siRNA\#2 targeting circRNA-MYLK. Moreover, the influences of the siRNA\# 1 or siRNA\#2 on the p-MEK and p-ERK, as well as the p/t-MEK ( 0.38 or 0.64 times) and p/t-ERK ( 0.72 or 0.68 times) (both $p<0.001$, Fig. 6A, B) were disturbed by the miR-145-5p inhibitor with significant increase on these elements. Additionally, the productions of p-NF- $\kappa \mathrm{B}-\mathrm{p} 65$ (Fig. 6C) and $\mathrm{p} / \mathrm{t}-\mathrm{NF}-\mathrm{kB}-\mathrm{p} 65$ (49 or $38 \%$ ) were inhibited by the siRNA\#1 or siRNA\#2 (both $p<0.05$, Fig. 6D) but were enforced by the miR-145-5p inhibitor (1.17 or 1.23 times) $(p<0.01$ or $p<0.001)$. These data displayed that siRNA\# 1 and siRNA\#2 targeting circRNA-MYLK deactivated the MEK/ERK and NF- $\kappa B$ signaling cascades in the Hep-2 cell line by promoting miR-145-5p. We could guess that circRNA-MYLK had the potentials to trigger the signaling cascades by targeting miR-145-5p.

\section{Discussion}

We noticed that circRNA-MYLK was overexpressed in laryngocarcinoma tissues. What's more, the viability, invasion and migration as well as the MEK/ERK and NF- $\mathrm{KB}$ cascades were significantly reduced in the Hep- 2 cell line by siRNA\# 1 and siRNA\#2 targeting circRNA-MYLK. Furthermore, miR-145-5p was negatively modulated by circRNA-MYLK. The anti-tumor impacts of siRNA\# 1 and siRNA\#2 were completely eliminated by miR-145-5p inhibitor, indicating a target relationship between the circRNA-MYLK and the miR-145-5p.
A

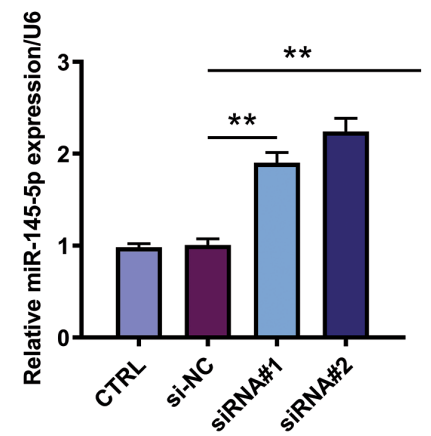

B

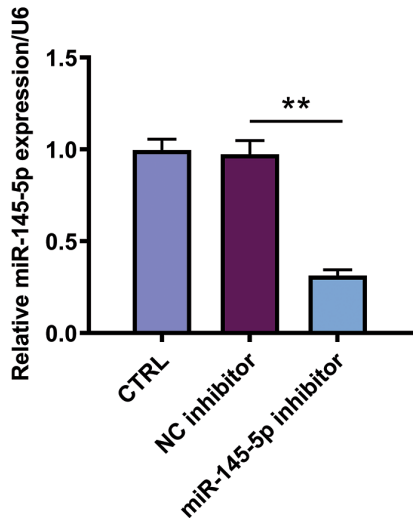

Figure 4. Knockdown of circRNA-MYLK induced high expression of miR-145-5p. qRT-PCR was conducted to examine the expression level of miR-145-5p after the transfection of siRNA\#1 and siRNA\#2 (A), and after the transfection of miR-145-5p inhibitor (B). ${ }^{* *} p<0.01$. miR-145$5 p$, microRNA-145-5p. For more abbreviations, see Fig. 1. 
A

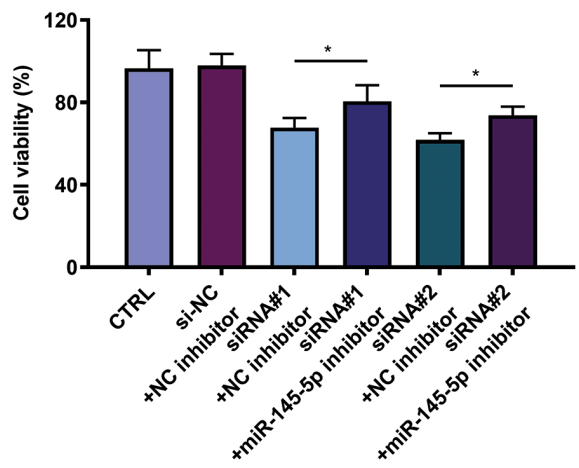

C

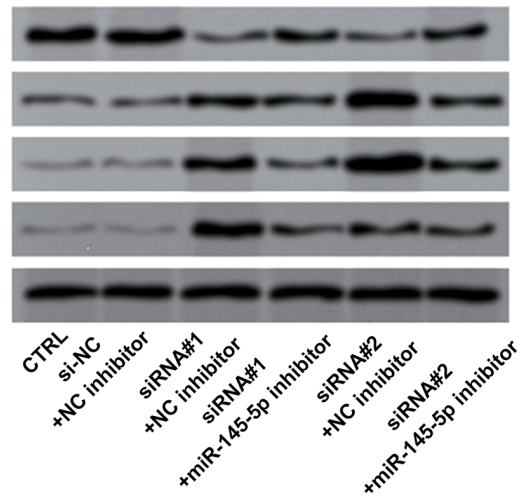

E

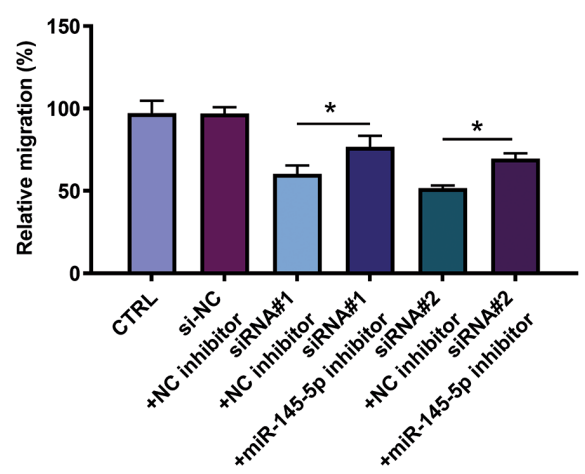

G

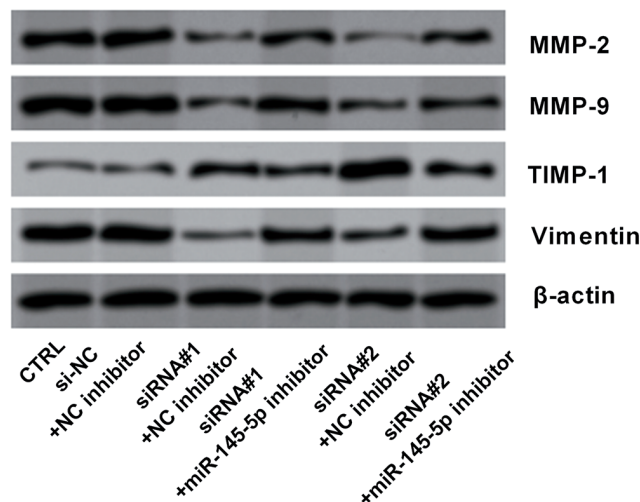

B

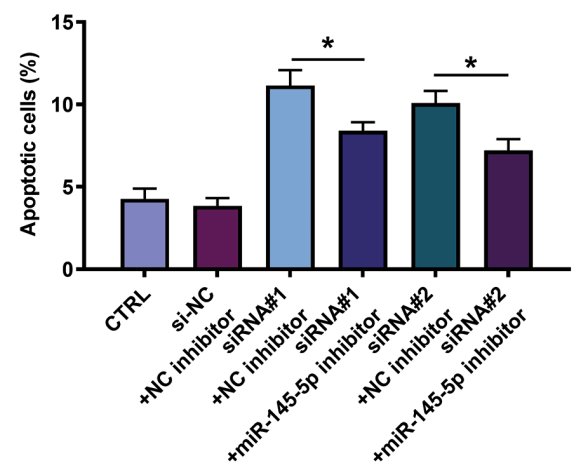

D

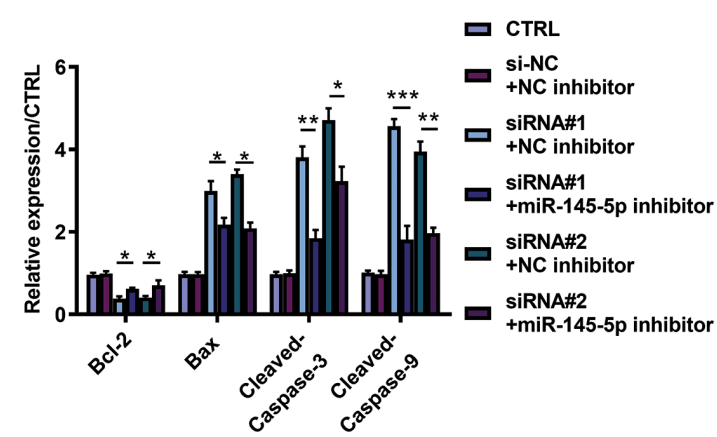

F

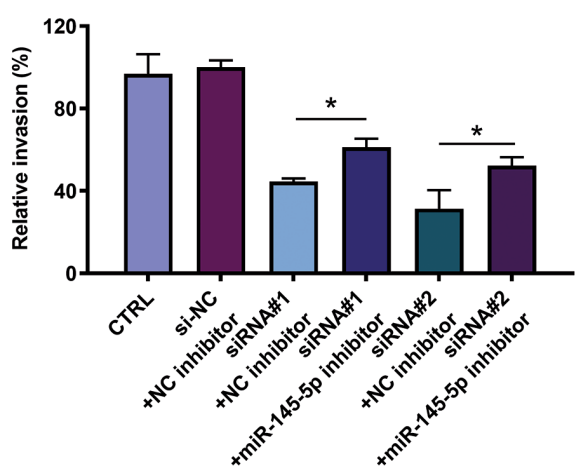

H

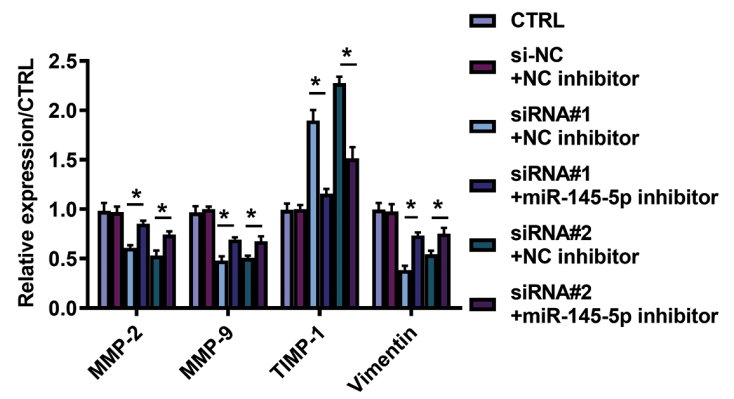

Figure 5. Silencing circRNA-MYLK induced apoptosis and inhibitions of cell viability, migration and invasion via up-regulating miR145-5p. After the transfections of relative elements, cell viability (A) and the apoptotic rate (B) was tested by CCK-8 or Annexin V-FITC/ PI-flow cytometer; the migration rate and invasion rate were detected with chamber assays $(\mathbf{E}, \mathbf{F})$; the apoptosis-related proteins $(\mathbf{C}$, D), and the migration and invasion proteins $(\mathbf{G}, \mathbf{H})$ were examined by Western blot. ${ }^{\star} p<0.05,{ }^{* \star} p<0.01,{ }^{* * *} p<0.001$. miR-145-5p, microRNA-145-5p; FITC, fluorescein isothiocyanate; MMP 2/9, matrix metalloprotein-2/9; TIMP, tissue inhibitor of metalloprotease 1; PI, propidium iodide; CCK-8, cell counting kit-8. For more abbreviations, see Fig. 1. 
A

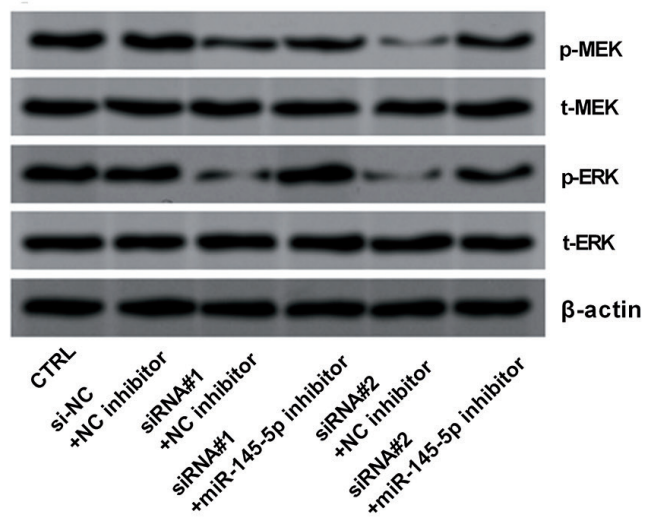

C

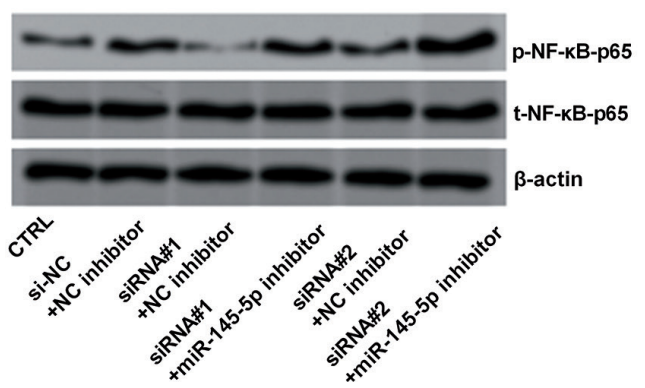

B

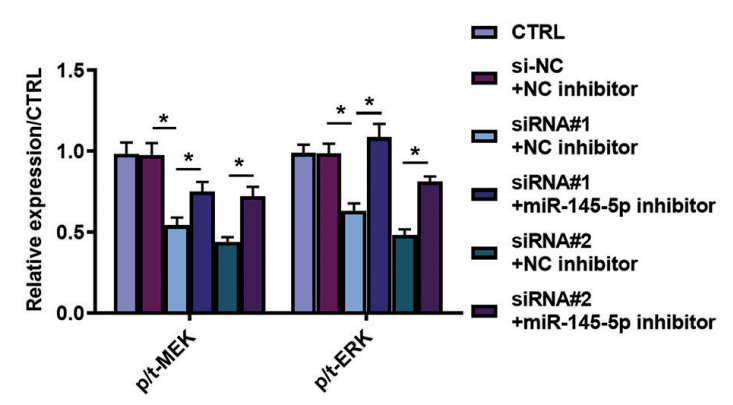

D

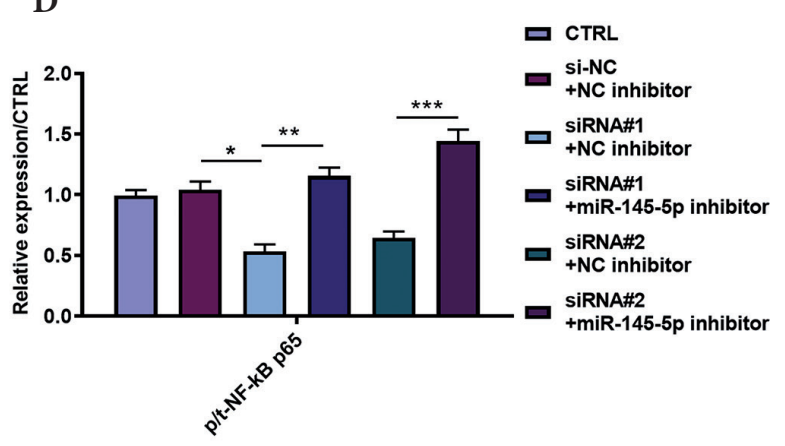

Figure 6. circRNA-MYLK silencing blocked MEK/ERK and NF- $\kappa B$ cascades by up-regulating miR-145-5p. After the transfections of relative elements, the p-MEK/ERK (A), p/t-MEK/ERK (B), p-NF-kB-p65 (C) and p/t-NF-kB-p65 (D) were identified with Western blot. ${ }^{\star} p<0.05,{ }^{* *} p<0.01,{ }^{* * *} p<0.001$. miR-145-5p, microRNA-145-5p; MEK, MAPK/ERK kinase; MAPK, mitogen-activated protein kinase; ERK, extracellular regulated protein kinases; NF- $\mathrm{kB}-$ p65, nuclear factor $\kappa B-p 65$. For more abbreviations, see Fig. 1.

CircRNAs are ncRNAs that have a stable and conserved loop structure ( $\mathrm{Li}$ et al. 2015). Copious evidences have demonstrated that circRNAs are significantly involved in the progressions of tumors. What's more, circRNA-MYLK is beneficial to the survival of prostate cancer (PCa) cells by adjusting the proliferation and migration procedures, representing a relationship between the circRNA-MYLK and tumors. Our outcomes observed that deviant expression of circRNA-MYLK was related to the tumorigenesis of laryngocarcinoma. CircRNA-MYLK silencing led to evident inhibition of cells survival. Among them, the converting of the apoptosis, migration and invasion is considered as the typical feature of cancer cells, while apoptosis is cell death response with unique morphological features and evasion of apoptosis represents one of the hallmarks of cancer (Pistritto et al. 2016). Just like what had been proved by Li et al. (2019), that knockdown of circRNA-MYLK significantly restrained HCC cells invasion and migration in Huh7 and Hep3B cells. In addition, Dai et al. (2018) observed abundant circRNA-MYLK in PCa samples and circRNA-MYLK was overexpressed to promote cells proliferation, invasion, and migration.

What's more, miR-145-5p is a member of the miRNAs family, and it has pivotal influences in adjusting cellular processes in diverse tumors (Mei et al. 2017; Wang et al. 2019), including LSCC. Zhang et al. found that miR-145$5 \mathrm{p}$ was connected with the suppression of LSCC (Gao et al. 2019). Out of question, an ingenious phenomenon was obtained in our investigation. The miR-145-5p, which was a downstream target of the circRNA-MYLK, was overexpressed to disturb the influences of siRNA\#1 and siRNA\#2 in Hep- 2 cell line. Just like that, Zhao's team turned out that plentiful circCEP128 modulated the miR-145-5p and the myeloid differentiation factor 88 (MyD88) to accelerate the progression of bladder cancer through MAPK cascade (Sun et al. 2019).

Being involved in the pathogenesis and development processes, ERK acts in various cancers such as prostate cancer, kidney cancer, colon cancer and esophageal squamous cell carcinoma (ESCC) (Wang et al. 2017). Moreover, the MEK- 
ERK cascade was conductive to the growth of laryngeal cancer cells, which was simulated by the phosphotyrosine phosphatase 2 (SHP2) (Gu et al. 2014). SH3-domain GRB2like 2 (SH3GL2) was implicated in the modulation of apoptosis through the MEK-ERK signaling cascade via regulating epidermal growth factor receptor (EGFR) in the Hep2 cell line (Shang et al. 2010). Besides, it had been revealed that the carboplatin combined with the 9-hydroxypheophorbide $a$ to prevent the metastasis of laryngeal cancer due to the MEK/ ERK pathway (Mao et al. 2016). It has been investigated that the NF- $\kappa \mathrm{B}$ is a major factor that was able to control tumor apoptosis, angiogenesis and invasiveness (Fan et al. 2013). Moreover, NF- $\kappa \mathrm{B}$ is quite implicated in the development of laryngocarcinoma. For instance, it had been revealed that testes-specific protease 50 (TSP50) silencing repressed the proliferation but induced apoptosis in HEp2 cell line in a NF- $\kappa \mathrm{B}$-mediated signaling transduction way (Zhang et al. 2013). Besides, it has been demonstrated that NF- $\kappa B$ signaling pathway got involved in the apoptosis modulation in laryngocarcinoma cell lines caused by the thrichosanthin (TCS) (Zhang et al. 2013).

These conclusions told information that MEK/ERK and $\mathrm{NF}-\kappa \mathrm{B}$ signaling cascades might have pivotal potentials to regulate the expansion of laryngocarcinoma. We observed in our study that knockdown of circRNA-MYLK targeted the miR-145-5p to block the MEK/ERK and NF- $\kappa B$ cascades. We could assume that circRNA-MYLK silencing stimulated the miR-145-5p to exert anti-tumor effects, which was relying on the blocks MEK/ERK and NF- $\kappa B$ pathways. This result could be partly confirmed by a few previous investigations. For example, the circRNA-MYLK was highly expressed to promote cell growth and metastasis by binding to miR-29a through the activation of relative signaling pathways in bladder carcinoma (Zhong et al. 2017). This conclusion revealed that circRNA-MYLK probably functioned in cancers or tumors by controlling signaling pathways. Based on that, circRNAMYLK probably was a crucial factor for signaling cascades controlling in many tumors, albeit enough evidences had not been accessed. Additionally, miR-145-5p participated in the regulation of the MEK/ERK pathway and thus mediated the protective effects of GEN on H9c2 cell line (Su et al. 2018). miR-145-5p displayed suppressive influences in esophageal squamous cell carcinoma (ESCC), and the expression level of miR-145-5p in cells was tightly connected with the sensitivity of NF- $\kappa$ B cascade (Mei et al. 2017).

Above all, we found that circRNA-MYLK silencing might exhibit anti-tumor roles in the Hep-2 cell by enhancing miR-145-5p through the blocks of MEK/ERK and NF- $\kappa B$ pathways.

Availability of data and materials. The datasets used and/or analyzed during the current study are available from the corresponding author on reasonable request.
Conflict of interest. The authors declare that they have no competing interests.

Fundings. This research did not receive any specific grant from funding agencies in the public, commercial, or not-for-profit sectors.

Authors' contributions. Conceived and designed the experiments: Xiaoyan Sun. Performed the experiments and analyzed the data: Yao Chen, Yanmei Wang, Congcong Li, Xuechang Li, Tiejun Yuan, Shuqin Yang. Wrote the manuscript: Yao Chen, Xiaoyan Sun.

\section{References}

Chen J, Li Y, Zheng Q, Bao C, He J, Chen B, Lyu D, Zheng B, Xu Y, Long Z, et al. (2017): Circular RNA profile identifies circPVT1 as a proliferative factor and prognostic marker in gastric cancer. Cancer Lett. 388, 208-219

https://doi.org/10.1016/j.canlet.2016.12.006

Chen JY, Hour TC, Yang SF, Chien CY, Chen HR, Tsai KL, Ko JY, Wang LF (2015): Autophagy is deficient in nasal polyps: implications for the pathogenesis of the disease. Int. Forum Allergy Rhinol. 5, 119-123 https://doi.org/10.1002/alr.21456

Chen L, Liu S, Li K, Qi J, Liu C, Zong L, Zhang Y, Zhao J, Zhai X, Li J, et al. (2018): Evaluation of microRNA expression profiling in highly metastatic laryngocarcinoma cells. Acta Otolaryngol. 138, 1105-1111 https://doi.org/10.1080/00016489.2018.1508887

Chen X, Zhu H, Ye W, Cui Y, Chen M (2019): MicroRNA29a enhances cisplatin sensitivity in nonsmall cell lung cancer through the regulation of REV3L. Mol. Med. Rep. 19, 831-840 https://doi.org/10.3892/mmr.2018.9723

Dai Y, Li D, Chen X, Tan X, Gu J, Chen M, Zhang X (2018): Circular RNA myosin light chain kinase (MYLK) promotes prostate cancer progression through modulating Mir-29a expression. Med. Sci. Monit. 24, 3462-3471 https://doi.org/10.12659/MSM.908009

Fan X, Zhao Y (2019): miR-451a inhibits cancer growth, epithelialmesenchymal transition and induces apoptosis in papillary thyroid cancer by targeting PSMB8. J. Cell. Mol. Med. 23, $8067-8075$

https://doi.org/10.1111/jcmm.14673

Fan Y, Mao R, Yang J (2013): NF-kappaB and STAT3 signaling pathways collaboratively link inflammation to cancer. Protein Cell 4, 176-185

https://doi.org/10.1007/s13238-013-2084-3

Gao W, Zhang Y, Niu M, Bo Y, Li H, Xue X, Lu Y, Zheng X, Tang Y, Cui J, et al. (2019): Identification of miR-145-5p-centered competing endogenous RNA network in laryngeal squamous cell carcinoma. Proteomics 19, e1900020-1900020 https://doi.org/10.1002/pmic.201900020

Gu J, Han T, Ma RH, Zhu YL, Jia YN, Du JJ, Chen Y, Jiang XJ, Xie XD, Guo X (2014): SHP2 promotes laryngeal cancer growth through the Ras/Raf/Mek/Erk pathway and serves as a prognostic indicator for laryngeal cancer. Int. J. Oncol. 44, 481-490 https://doi.org/10.3892/ijo.2013.2191 
Lambert L, Fortin B, Soulieres D, Guertin L, Coulombe G, Charpentier D, Tabet JC, Belair M, Khaouam N, Nguyen-Tan PF (2010): Organ preservation with concurrent chemoradiation for advanced laryngeal cancer: are we succeeding? Int. J. Radiat. Oncol. Biol. Phys. 76, 398-402 https://doi.org/10.1016/j.ijrobp.2009.01.058

Lampri ES, Chondrogiannis G, Ioachim E, Varouktsi A, Mitselou A, Galani A, Briassoulis E, Kanavaros P, Galani V (2015): Biomarkers of head and neck cancer, tools or a gordian knot? Int. J. Clin. Exp. Med. 8, 10340-10357

Li P, Chen S, Chen H, Mo X, Li T, Shao Y, Xiao B, Guo J (2015): Using circular RNA as a novel type of biomarker in the screening of gastric cancer. Clin. Chim. Acta 444, 132-136 https://doi.org/10.1016/j.cca.2015.02.018

Li P, Liu H, Wang Z, He F, Wang H, Shi Z, Yang A, Ye J (2016): MicroRNAs in laryngeal cancer: implications for diagnosis, prognosis and therapy. Am. J. Transl. Res. 8, 1935-1944

Li Z, Hu Y, Zeng Q, Wang H, Yan J, Li H, Yu Z (2019): Circular RNA MYLK promotes hepatocellular carcinoma progression by increasing Rab23 expression by sponging miR-362-3p. Cancer Cell Int. 19, 211 https://doi.org/10.1186/s12935-019-0926-7

Liu J, Sheng W, Xie Y, Shan Y, Miao J, Xiang J, Yang J (2010): The in vitro and in vivo antitumor activity of adenovirus-mediated interleukin-24 expression for laryngocarcinoma. Cancer Biother. Radiopharm. 25, 29-38 https://doi.org/10.1089/cbr.2009.0706

Mao W, Sun Y, Zhang H, Cao L, Wang J, He P (2016): A combined modality of carboplatin and photodynamic therapy suppresses epithelial-mesenchymal transition and matrix metalloproteinase-2 (MMP-2)/MMP-9 expression in HEp-2 human laryngeal cancer cells via ROS-mediated inhibition of MEK/ERK signalling pathway. Lasers Med. Sci. 31, 1697-1705 https://doi.org/10.1007/s10103-016-2040-6

Mei LL, Wang WJ, Qiu YT, Xie XF, Bai J, Shi ZZ (2017): miR-145$5 p$ suppresses tumor cell migration, invasion and epithelial to mesenchymal transition by regulating the Sp1/NF-kappaB signaling pathway in esophageal squamous cell carcinoma. Int. J. Mol. Sci. 18, 1833 https://doi.org/10.3390/ijms18091833

Miao S, Mao X, Pei R, Miao S, Xiang C, Lv Y, Yang X, Sun J, Jia S, Liu Y (2013): Antitumor activity of polysaccharides from Lepista sordida against laryngocarcinoma in vitro and in vivo. Int. J. Biol. Macromol. 60, 235-240 https://doi.org/10.1016/j.ijbiomac.2013.05.033

Pistritto G, Trisciuoglio D, Ceci C, Garufi A, D`Orazi G (2016): Apoptosis as anticancer mechanism: function and dysfunction of its modulators and targeted therapeutic strategies. Aging 8, 603-619 https://doi.org/10.18632/aging.100934

Shang C, Guo Y, Fu S, Fu W, Sun K (2010): SH3GL2 gene participates in MEK-ERK signal pathway partly by regulating EGFR in the laryngeal carcinoma cell line Hep2. Med. Sci. Monit. 16, Br168-173

Song L, Liu S, Yao H, Zhang L, Li Y, Xu D, Li Q (2019): MiR-362-3p is downregulated by promoter methylation and independently predicts shorter OS of cervical squamous cell carcinoma. Biomed. Pharmacother. 115, 108944-108944

https://doi.org/10.1016/j.biopha.2019.108944

Su Q, Yao J, Sheng C (2018): Geniposide Attenuates LPS-Induced Injury via Up-Regulation of miR-145 in H9c2 Cells. Inflammation 41, 1229-1237 https://doi.org/10.1007/s10753-018-0769-8

Sun M, Zhao W, Chen Z, Li M, Li S, Wu B, Bu R (2019): Circular RNA CEP128 promotes bladder cancer progression by regulating Mir-145-5p/Myd88 via MAPK signaling pathway. Int. J. Cancer 145, 2170-2181 https://doi.org/10.1002/ijc.32311

Wang B, Dong W, Li X (2019): miR-145-5p acts as a novel tumor suppressor in hepatocellular carcinoma through targeting RAB18. Technol. Cancer Res. Treat. 18, 1533033819850189 https://doi.org/10.1177/1533033819850189

Wang H, Zhang Y, Yun H, Chen S, Chen Y, Liu Z (2017): ERK expression and its correlation with STAT1 in esophageal squamous cell carcinoma. Oncotarget 8, 45249-45258 https://doi.org/10.18632/oncotarget.16902

Xuan L, Qu L, Zhou H, Wang P, Yu H, Wu T, Wang X, Li Q, Tian L, Liu M, Sun Y (2016): Circular RNA: a novel biomarker for progressive laryngeal cancer. Am. J. Transl. Res. 8, 932-939

Zhang D, Lu LM, Chen B, Li Q, Zhou J, Tao L (2013): Thrichosanthin enhances cisplatin-induced laryngocarcinoma apoptosis through inhibiting transcription factor nuclear factor kappaB. Zhonghua Er Bi Yan Hou Tou Jing Wai Ke Za Zhi 48, 322-328 (in Chinese)

Zhong Z, Huang M, Lv M, He Y, Duan C, Zhang L, Chen J (2017): Circular RNA MYLK as a competing endogenous RNA promotes bladder cancer progression through modulating VEGFA/ VEGFR2 signaling pathway. Cancer Lett. 403, 305-317 https://doi.org/10.1016/j.canlet.2017.06.027

Zhuang S, Liu F, Wu P (2019): Upregulation of long noncoding RNA TUG1 contributes to the development of laryngocarcinoma by targeting miR-145-5p/ROCK1 axis. J. Cell. Biochem. 120, 13392-13402 https://doi.org/10.1002/jcb.28614

Received: June 19, 2019

Final version accepted: December 6, 2019 